


\title{
PERFORMANCE CHARACTERISTICS OF PROXIMITY FOCUSED ULTRAVIOLET IMAGE CONVERTERS
}

\author{
by
}

Jack T. Williams and Walter A. Feibelman

Goddard Space Flight Center

National Aeronautics and Space Administration Greenbelt, Maryland

\begin{abstract}
Performance characteristics of Bendix type BX 8025-4522 proximity focused image tubes for ultraviolet to visible light conversion are presented. Quantum efficiency, resolution, background, geometric distortion and environmental test results are discussed. The converters use magnesium fluoride input windows with Cs-Te photocathodes, and P-II phosphors on fiber optic output windows.
\end{abstract}

INTRODUCTION

Proximity focused ultraviolet to visible light image converters have been evaluated for use in low light level UV imaging systems. These sensors are being developed for application in rocket and satelite astronomical observations. The performance characteristics which have been measured include those of the $\mathrm{UV}$ to visible converters alone as well as in conjunction with an SEC vidicon in a UV-TV system as shown in Fig. 1. This TV system utilizes Westinghouse type WX 32224 SEC vidicons and will be used as detectors for the echelle spectrograph cameras on the International Ultraviolet Explorer. This satellite is a joint UK-ESRO-US effort scheduled for launch in 1976 and is to be placed in a geosynchronous orbit to obtain ultraviolet astronomical spectra! (1) 
The Bendix BX 8025-4522 UV to visible converters are compact, low power, proximity focused imaging devices which supply both gain and UV to visible photon conversion. These tubes consist of five basic parts as shown in Fig. 2 . The input window is magnesium fluoride and was chosen over other. UV transmissive materials, such as lithium fluoride or sapphire, because of its relative hardness, stability in air without absorption, short wavelength cutoff below $120 \mathrm{~nm}$ and its resistance to radiation effects such as formation of color centers and scintillations, all of which are necessary in a successful rocket or satellite experiment. Cesium telluride was chosen for the photocathode because of its high response to $U V$ and lack of response to visible radiation, resulting in a useful sensitivity range from the input window cutoff near $115 \mathrm{~nm}$ to approximately $350 \mathrm{~nm}(2)$. The thin aluminum layer performs several functions: anode connection for the high voltage supply, white light rejection from the phosphor to a value of at least $10^{4}$, prevention of $\mathrm{UV}$ photons directly exciting the phosphor, and prevention of backscattering of light from the phosphor. The P-11 phosphor is a blue short persistence phosphor, the spectral response of which is well matched both to film and to the bi-alkali photocathode of the SEC vidicon to be used in the UV-TV system. The fiber optic output window provides an excellent flat display at the output and is ideally suited for detection by the eye as well as direct contact with film, eliminating the need for coupling lenses. The fiber optic window of the converter also permits direct face to face coupling to the fiber optic input window used on the SEC vidicon. The minimum high voltage potential applied between the photocathode and phosphor is determined by the kinetic energy needed for the photoelectrons to penetrate the aluminum layer. An upper limit of $6 \mathrm{KV}$ on the tubes tested was determined by the onset of flares at the output and by the potential separation of the phosphor layer 
from the output fiber optic due to the intense field created in the narrow spacing between the photocathode and aluminum layer. The performance of the UV to visible converters was evaluated in terms of quantum efficiency, resolution as a function of wavelength, background and gain. These parameters are discussed both for the converter alone and for the converter coupled to a TV camera tube operating in an analog mode at thirty frames per second.

QUANTUM EFFICIENCY

Quantum efficiency measurements were obtained using a continuous flow hydrogen source on a McPherson model $225 \mathrm{UV}$ monochromator equipped with a differential pumping system. Measurements were made by operating the tube as a non-imaging diode at about 20 separate wavelengths covering the range from $115 \mathrm{~nm}$ to $350 \mathrm{~nm}$. Absolute values were obtained by comparison with data obtained with an EMR model 543P-09 00 diode phototube with calibration traceable to the US National Bureau of standards. In Fig. 3 is shown the average results of measurements made on the Cs-Te photocathodes of 6 separate converters. The center (solid) curve represents the average of all 6 photocathode measurements, while the two bracketing curves are plots of the maximum (upper curve) and minumum (lower curve) ialues measured at each wavelength. These curves are typical in shape and peak efficiency for Cs-Te photocathodes currently available from Bendix. They show an improvement over earlier Cs-Te cathodes by Bendix as well as other manufacturers, as shown in Fig. 4. Comparison of Fig. 3 and 4 indicates that the improvement consists of a "squaring" of the peak at a higher value and a sharper cutoff at the long wavelength end.

RESOLUTION

Resolution is measured in terms of a square wave modulation transfer function (MTF) curve on which the percent contrast of the output image is plotted against the spatial frequency of a 100 percent contrast square wave bar pattern. The bar 
patterns used were nickel evaporated on UV transmitting magnesium fluoride disks and were manufactured on special order by Metrographics, Inc. MTF measurements were obtained by a two-step process. First, the MTF of a Westinghouse WL 30893 SEC vidicon was measured by placing the bar pattern on the fiber optic input window and monitoring a single TV scan line at the center of the video output. Second, using Eastman Kodak HE-79 optical cement, the Bendix converter was coupled to the vidicon, anci the MTF of the coupled converter/vidicon combination was measured using the bar pattern on the converter input window and illuminating it with $U V$ radiation. The converter MTF values were then obtained by dividing the MTF of the converter/vidicon combination by the MTF of the vidicon alone. In this manner we were able to obtain MTF curves at $121.6 \mathrm{~nm}, 160.8 \mathrm{~nm}, 200 \mathrm{~nm}$ and $250 \mathrm{~nm}$. This method was chosen because we were interested primarily in the characteristics of the converter/vidicon combination. Therefore, it should be noted that the resultant curves for the converter alone include coupling factors (estinated to be about 0.95 ) between the converter and vidicon. Also the values shown assume a square wave output from the converter to the vidicon. Comparisons with sine wave MTF values calculated after the manner of Johnson (3) indicate that the error is within the experimental limits and on the negative side. Thus, the measurements are considered only good minimum values of the converter MTF. MTF curves for the vidicon alone as well as the converter/ vidicon combination at the previously mentioned wavelengths is shown in Fig. 5. The resultant converter MTF curves are shown in Fig. 6. A sharp decrease in resolution for the converters is evident at $160 \mathrm{~nm}$ relative to $121.6 \mathrm{~nm}$ and $250 \mathrm{~nm}$. Investigation into the causes of this phenomenon shown in Fig. 7 were carried out jointly by the IUE scientific team under Dr. A. Boksenberg at University College London, and at Goddard Space Flight Center. The explanation of this decrease in resolution, as given in the IUE Scientific Team Report ${ }^{(4)}$ is best understood 
using the computations based on the work of Needham and Thumwood (5) and Grant ${ }^{(6)}$ to obtain the theoretical performance of proximity focused image converters in which photoelectrons leaving the photocathode are assumed to begin with a Maxwellian distribution in energy and a Lambertian distribution in the direction of the velocity vectors. Allowing for the idealized assumptions of the energy distribution, the resulting values of most probable energy are in good agreement with published data for Cs-Te above $170 \mathrm{~nm}$ and the trend below $170 \mathrm{~nm}$ conforms to the production of holeelectron pairs in the semiconductor photocathode. The explanation of hole-electron pair production ${ }^{(8)}$ can be found in the fact that for a material like Cs-Te, if the photon energy is less than or equal to twice the bandgap, an excited electron will lose little energy before escaping from the photocathode and its energy in vacuum can be relatively large. However, when the photon energy is greater than twice the bandgap, the primary electron can excite secondary electrons and in the process lose most of its kinetic energy. Since resolution decreases as the kinetic energy of the photoelectron increases, the resolution should decrease monotonically with increasing photon energy until the threshold for pair production is reached; then the resolution should improve as the photon energy increases further. The bandgap for $\mathrm{Cs}-\mathrm{Te}$ is about $3.5 \mathrm{eV}$, so the worst resolution should occur at about $170 \mathrm{~nm}$. Examination of Fig. 7 shows that this is exactly what happens. Because of this phenomenon, the theoretically attainable resolution of a proximity focused device is dependent on the photocathode material, the proximity spacing, the high voltage potential, and the photon energy。: To increase resolution there are four possibilities: (1) increase high voltage, (2) decrease the proximity spacing, (3) change internal characteristics of the converter, and (4). change photocathode materials. This last possibility for increasing resolution 
(i.e. changing cathode material) is not useful in our application, where sensitivity is required below $300 \mathrm{~nm}$, coupled with high rejection of visible light.

Maximum resolution $(5)$ attainable is $R=2 \mathrm{~d}\left(\frac{\mathrm{w}}{\mathrm{e}^{V_{a}}}\right)^{1 / 2}$ where

$d$ is the proximity spacing, $w$ is the photon energy, e is charge of an electron and $\mathrm{V}_{\mathrm{a}}$ is the applied voltage. Thus, we see that resolution is most sensitive to changes in spacing and secondly to changes in applied voltage. The other items are essentially limited by the adherence of the aluminum and phosphor layers. This means that highest applied voltage and narrowest spacing are determined by the highest field that the aluminum and phosphor layers can withstand without being pulled off the output window.

An additional limitation on the resolution is due to internal halation effects. These effects were evaluated by monitoring the output image of the tube with the input window perpendicular to a very small beam of parallel $W$ light and then comparing it with the output when the tube was tilted at an angle of approximately $30^{\circ}$ to the same beam, $f_{1} 8$. The results indicate that UV light transmitted through the input window and photocathode strikes the aluminum layer and is reflected back to the photocathode where additional photoelectrons are released. These electrons cause a secondary or ghost image, to be produced at the phosphor thus decreasing the contrast or MTF of the tube. Actual measurement of the displacement of a $100 \mathrm{\mu m}$ pinhole (Fig. 9) are within $0.1 \mathrm{~mm}$ of values predicted by calculations and the secondary spot has an intensity approximately 10 percent of the primary image. Measurement at a spatial frequency of $4 \mathrm{lp} / \mathrm{mm}$ utilizing the same tilting procedure show an 
increase in MTF of approximately 50 percent when the tube is tilted. The effects of depositing blackened aluminum or carbon on the aluminum layer are being investigated in an effort to eliminate the reflections.

PHOTON GAIN

Photon gain was measured in terms of the number of visible photons at the output for each oV photon incident on the faceplate. The photon gain is defined $(9,10)$ as $G=Q(\lambda) x V e x y$ where $Q(\lambda)$ is the quantum efficiency in electrons per photon at wavelength $\lambda, \mathrm{v}_{\mathrm{e}}$ is the effective phosphor voltage (i.e. total voltage drop minus voltage required for penetration of the aluminum layer) and $\gamma(9,10,11)$ is the phosphor integral efficiency in photons per electron volt. Using this equation we find that for the Cs-Te converter one can expect peak photon gains of approximately 20. Gain measurements made on a typical converter are presented in Fig. 10 together with predicted values as a function of wavelength.

BACKGROUND

Background is dark current output of the tube with no photons incident on the photocathode. In applications where long time exposures are to be made the dark current is of utmost importance, because as exposure or integration time increase the background level will increase and eventually determine the lipper limit of exposure time. The background noise of these converters was measured by detecting the output photon dark current from the P-Il phosphor with a calibrated photomultiplier tube at the output fiber optic faceplate. Measured values of background for $6 \mathrm{Cs}-\mathrm{Te}$ converters cover the range from $4 \times 10^{-12} \mathrm{~W} \mathrm{~cm}^{-2}$ to $5 \times 10^{-14} \mathrm{~W}-\mathrm{cm}^{-2}$. These values, however, are averaged over the total output area. When the output is recorded on photographic film (Fig. 19), it becomes apparent that the background is not uniformly distributed at the output but rather is concentrated in one or two small points or flares, 
with the remainder of the output having background levels which are much lower. Indeed, tests with the flares masked show the background levels for the remaining area of the tube to be less than $1 \times 10^{-15} \mathrm{~W}_{-}^{-} \mathrm{cm}^{-2}$. A second source of background is visible light transmitted through pinholes in the aluminum layer, causing stray outputs. Pinholes in a typical converter illuminated with white light are shown in Fig. 12. The average number of pinholes observed was 38 , concentrated mostly at the edges. This problem can be entirely eliminated by increasing the thickness of the aluminum layer and by going to a larger format tube in which only the central portion is used for imaging. DISTORTION

Except for edge effects, distortion within a proximity focused device in theory should be minimal. Non-uniformities in phosphor and aluminum deposition, however, can result in geometric and resolution distortion. Tests using uniform bar patterns covering the entire active area of the tube to within $0.5 \mathrm{~mm}$ of the edge reveal no geometric distortion over the imaging area. Variations of $\sim_{1}$ percent between center and eage resolution measurements were observed. These variations are almost at the limit of experimental accuracy, suggesting that the effects are negligible.

ENVIRONMENTAL TESTS

A typlcal converter was coupled to an SEC vidicon camera tube and subjected to both random and sine vibrations along all three axes of the assembly. In addition, the converter/vidicon assembly was given a shock along two axes, one along the longitudinal axis and the second perpendicular to the first. The vibration levels are summarized in the table shown in Fig. 13 and the shock spectrum is shown in Fig. 14. Performance evaluations made before and after each type of vibration test revealed no detectable change in the number of blemishes on the tubes, background levels, resolution or quantum efficiency. 
Laboratory evaluation of Bendix UV proximity focused inage converters have demonstrated that these devices, coupled either to photographic or TV readout, are suitable for application in UV astronomy rocket and satelite systems. They should also prove useful for ground-based laboratory studies, such as UV spectroscopy, plasma physics and medical problems, either in conjunction with TV cameras or with photographic techniques. ACKNOWLEDGEMENTS

We thank Dr. A. Boggess III for guidance and valuable discussions during this program. We also thank Dr. W. E. Spicer for information on the bandgap behavior of Cs-Te photocathodes, as well as numerous other people in the UK and at Goddard who have contributed to this work. 


\section{REFERENCES}

1. Sturgell, C., Williams, J. T., Feibelman, W. A., Boksenberg, A., Anderson, B. E., McKrell, G. E. and Jones T. J. I. "Application of New Television Detectors in an Astronomical Satellite", Advances in Electronics and Electron Physics, 33B, Academic Press, New York, (1972)。

2. Sommer, A. H., "Photoemissive Materials", J. Wiley and Sons, New York (1968).

3. Johnson, C. B., "Classification of Electron-Optical Device Modulation Transfer Functions", Advances in Electronics and Electron Physics, 33B, Academic Press, New York, (1972).

4. UK Scientific Team, University College London, SAS-D Tech Note No. 6, (1972).

5. Needham, M. J. and Thumwood, R. F., "A Proximity Focused Image Tube", Adv, E。 E. P., 28A, 129, Academic Press, New York, (1969).

6. Grant, J., Proc. Inst. Electr. Electronic Engrs., 54, 801, (1966).

7. Taft, E. and Apker, L., J.O.S.A., 43, 81, (1953).

8. Spicer, W. L., private communication, (1971).

9. Owen, L. D., "The Proximity Focused Image Intensifier", Bendix Tech. Memo EOTM 7201, (Jan. 1972).

10. Owen, L. D., "Some Characteristics of Phosphors, Photocathodes and Proximity Focused Image Intensifiers", Bendix Tech. Memo EOTM 108, (1971).

11. Eberhardt, E. H., "Source-Detector Spectral Matching Factors", Applied Optics, 7,2037 , (1968). 


\section{FIGURE CAPTIONS}

Fig. 1 - Satellite instrumentation showing (a) 2 short wavelength UV-TV cameras, (b) 2 long wavelengths UV-TV cameras, (c) 2 white light acquisition TV cameras.

Fig. 2 - Cross section of Bendix proximity focussed image converters.

Fig. 3 - Measured average values of quantum efficiency for six converters (solid line) and maximum and minimum values (dashed line) for the region $115 \mathrm{~nm}$ to $350 \mathrm{~nm}$. The actual values for quantum efficiency lie about $10 \%$ below the measured values, due to internal reflections of UV photons that penetrated the photocathode and were reflected by the aluminum layer, thus striking the cathode again.

Fig. 4 - Typical quantum efficiency vs. wavelength curve for older Cs-Te photocathode.

Fig. 5 - Modulation transfer functions for white light SEC vidicon alone and for converter/SEC combination at four UV. wavelengths.

Fig. 6 - Modulation transfer functions for UV converter alone, deduced from Fig. 5 .

Fig. 7 - Average value of MTF for 6 converters without the SEC as a function of wavelength at a spatial frequency of 5 cycles $/ \mathrm{mm}$.

Fig. 8 - a. UV radiation entering perpendicularly to faceplate, resulting in diffuse image around primary image.

b. UV radiation entering at an angle $30^{\circ}$ to perpendicular, causing secondary image to be displaced from primary image. Intensity of secondary image is approximately $10 \%$ of primary.

Fig. 9 - Secondary image of a $100 \mu m$ pinhole displaced from primary image when tube is illuminated by a parallel beam of UV radiation incident $30^{\circ}$ from perpendicular to faceplate.

Fig. 10 - Predicted and measured values of photon gain. Predicted and measured values of photon gain. This curve is based on the quantum efficiency for a single converter and shows a larger peak near 3000A than the smoothed average curve for 6 converters of Fig. 3 . 
Fig. 11 - Photographic record of flares on output of converter. Fig. 12 - Typical pinhole pattern when converter is flooded by white light.

Fig. 13 - Table of vibration levels.

Fig. 14 - Shock spectrum. 







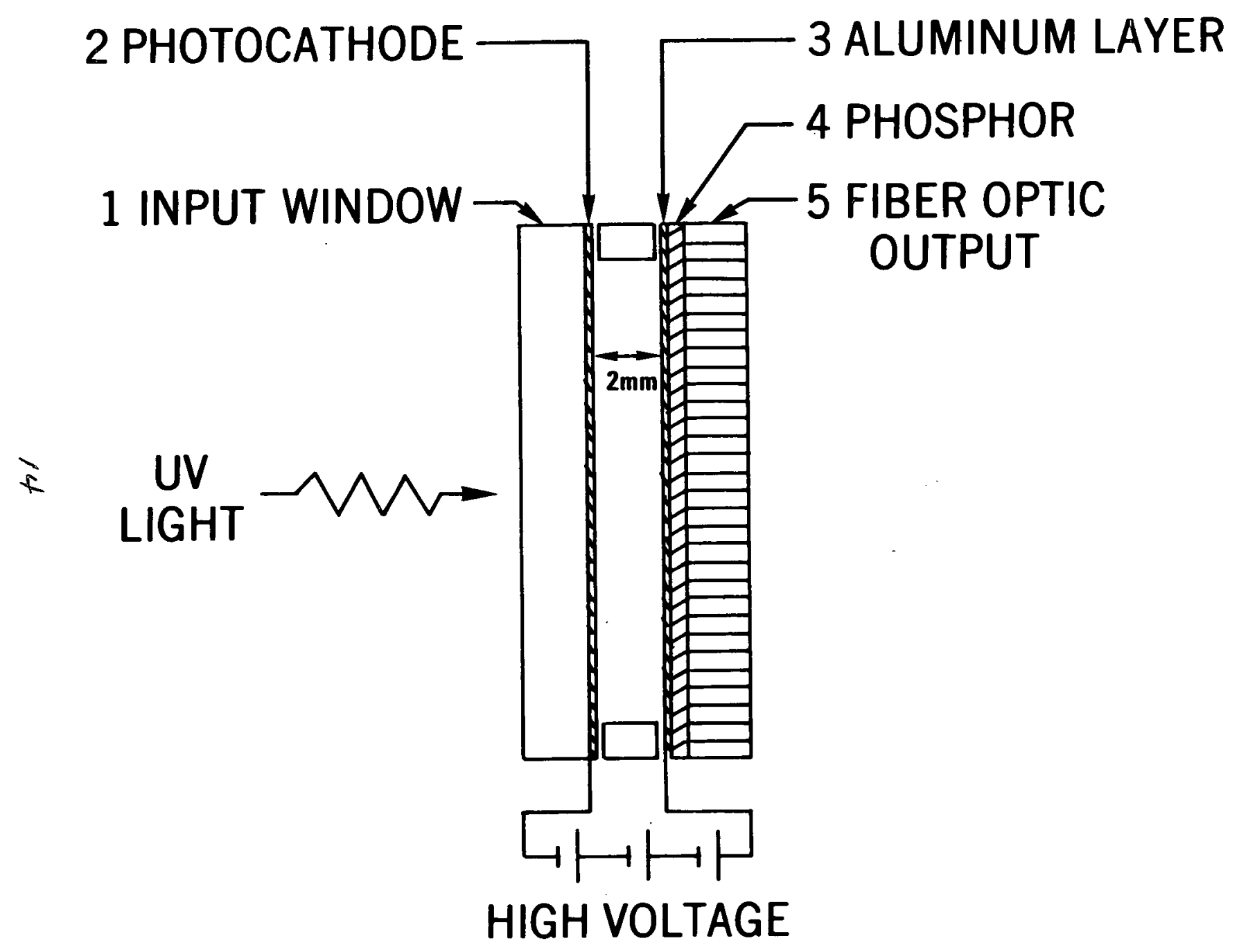




\section{AVERAGE QUANTUM EFFICIENCY}

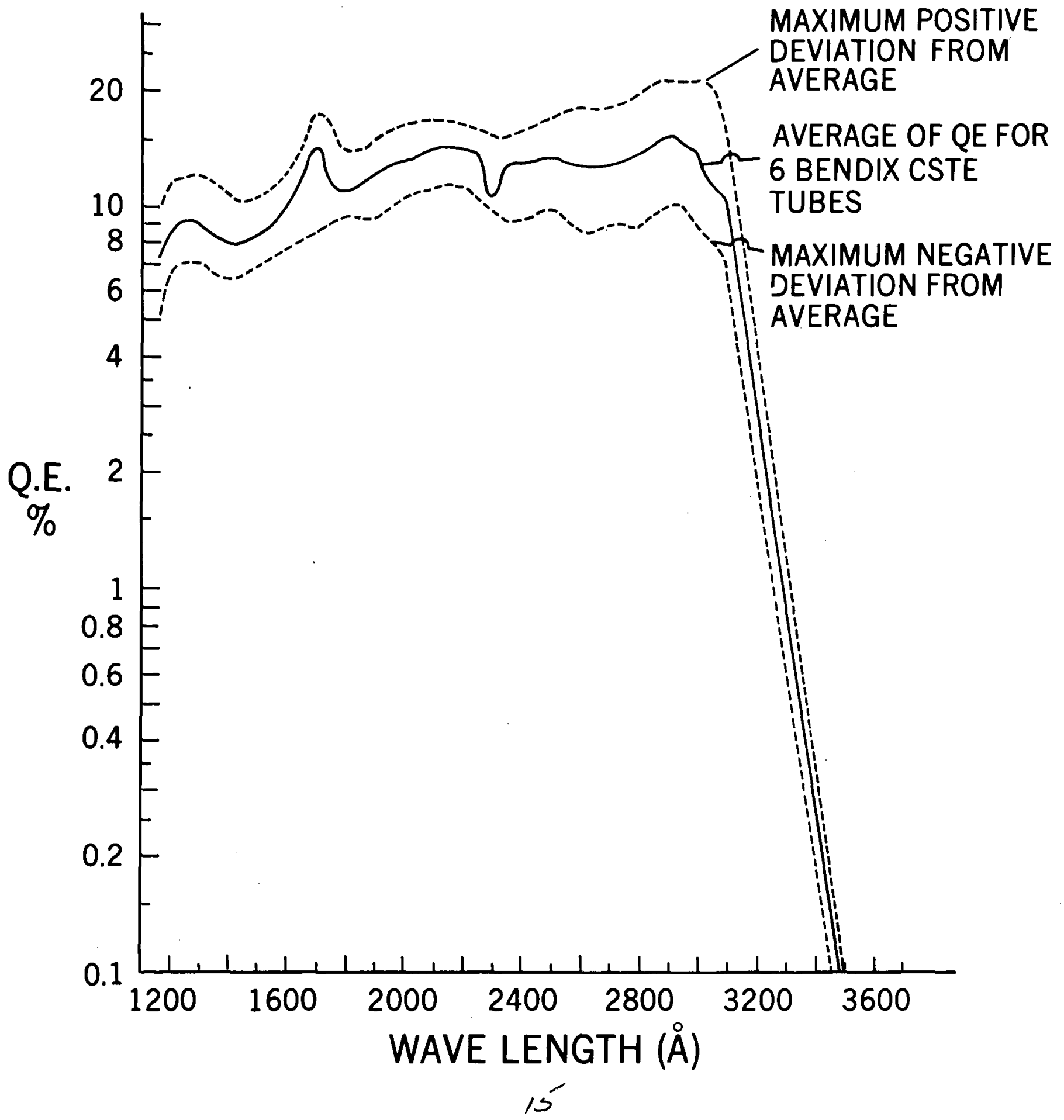




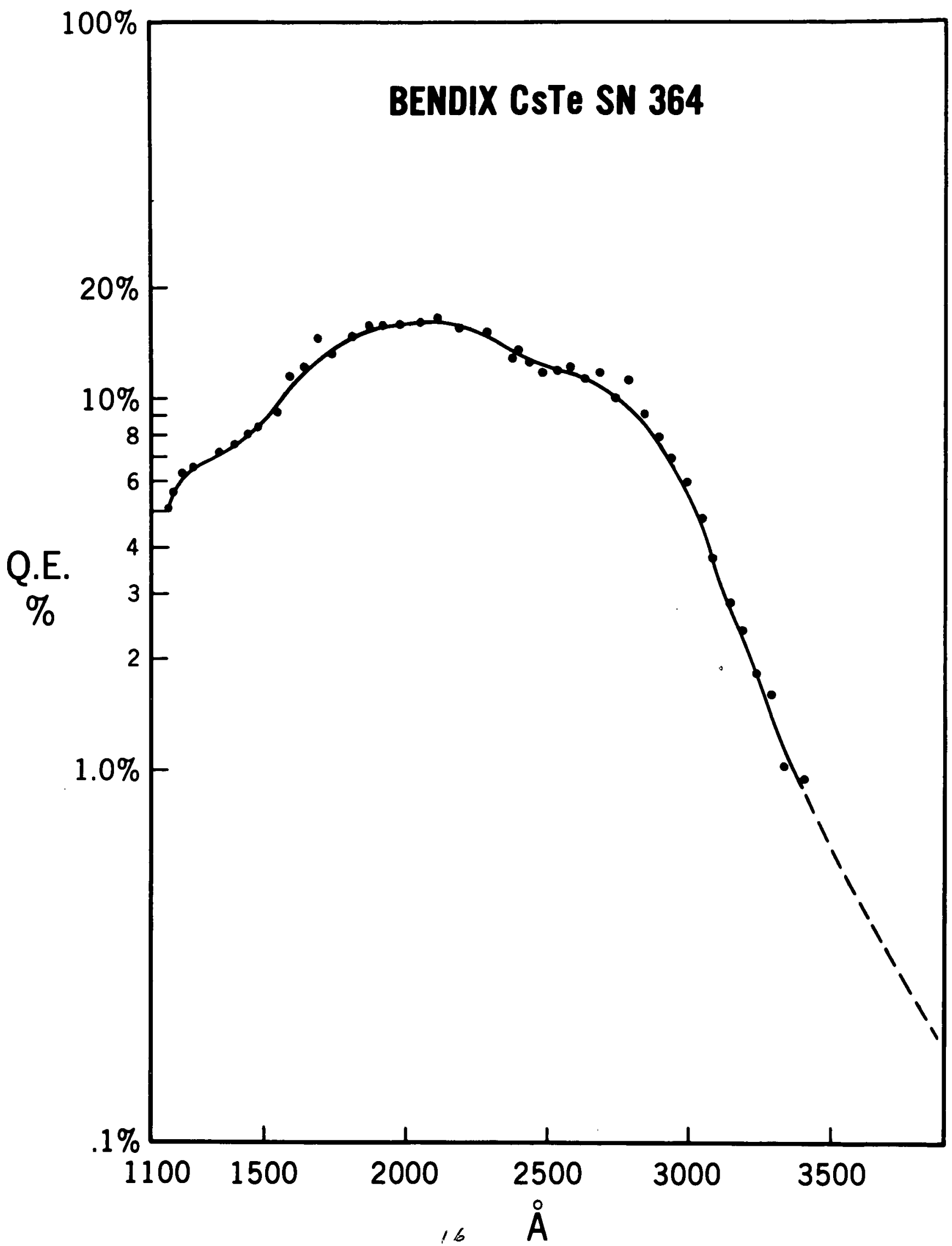




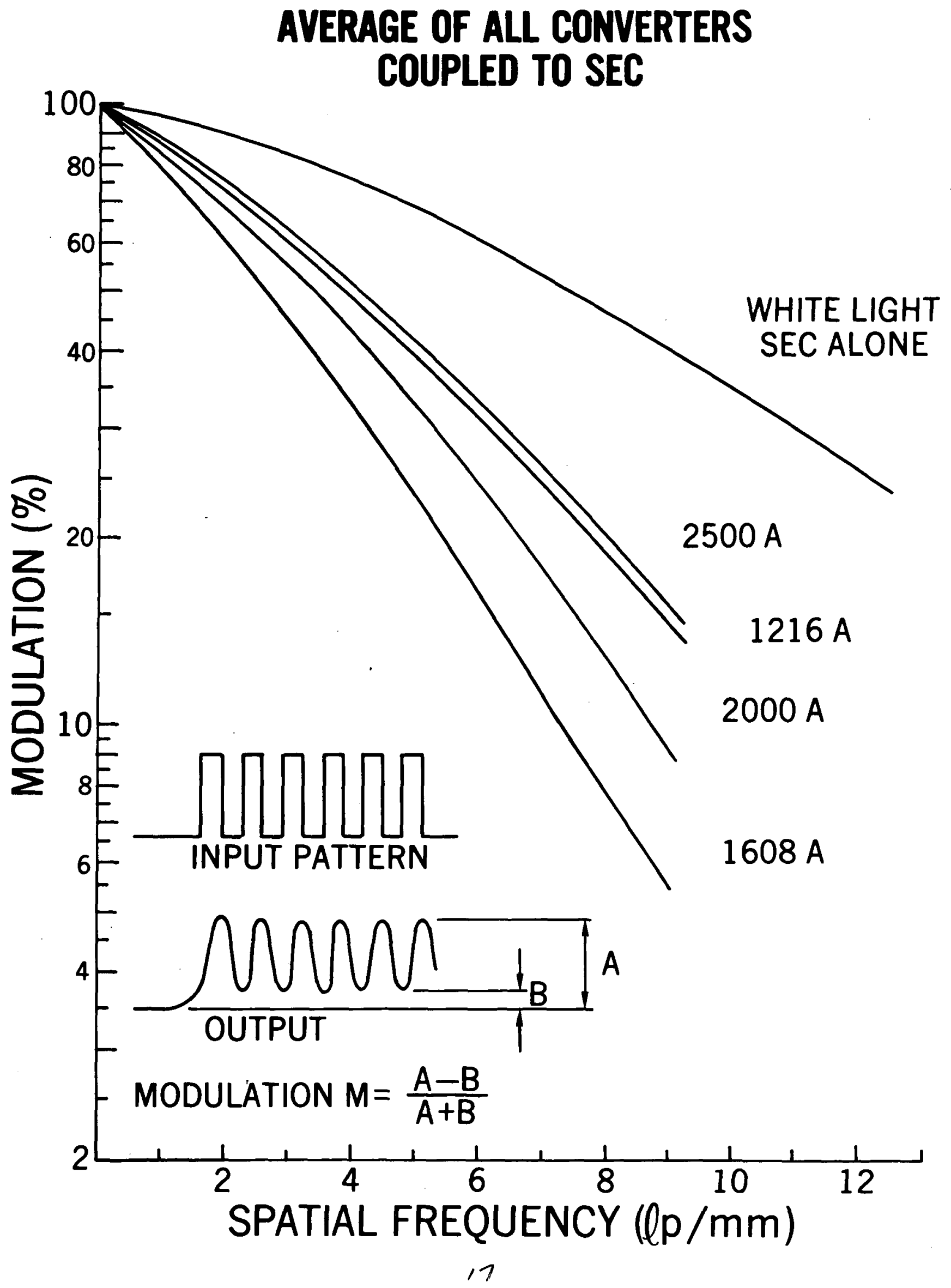




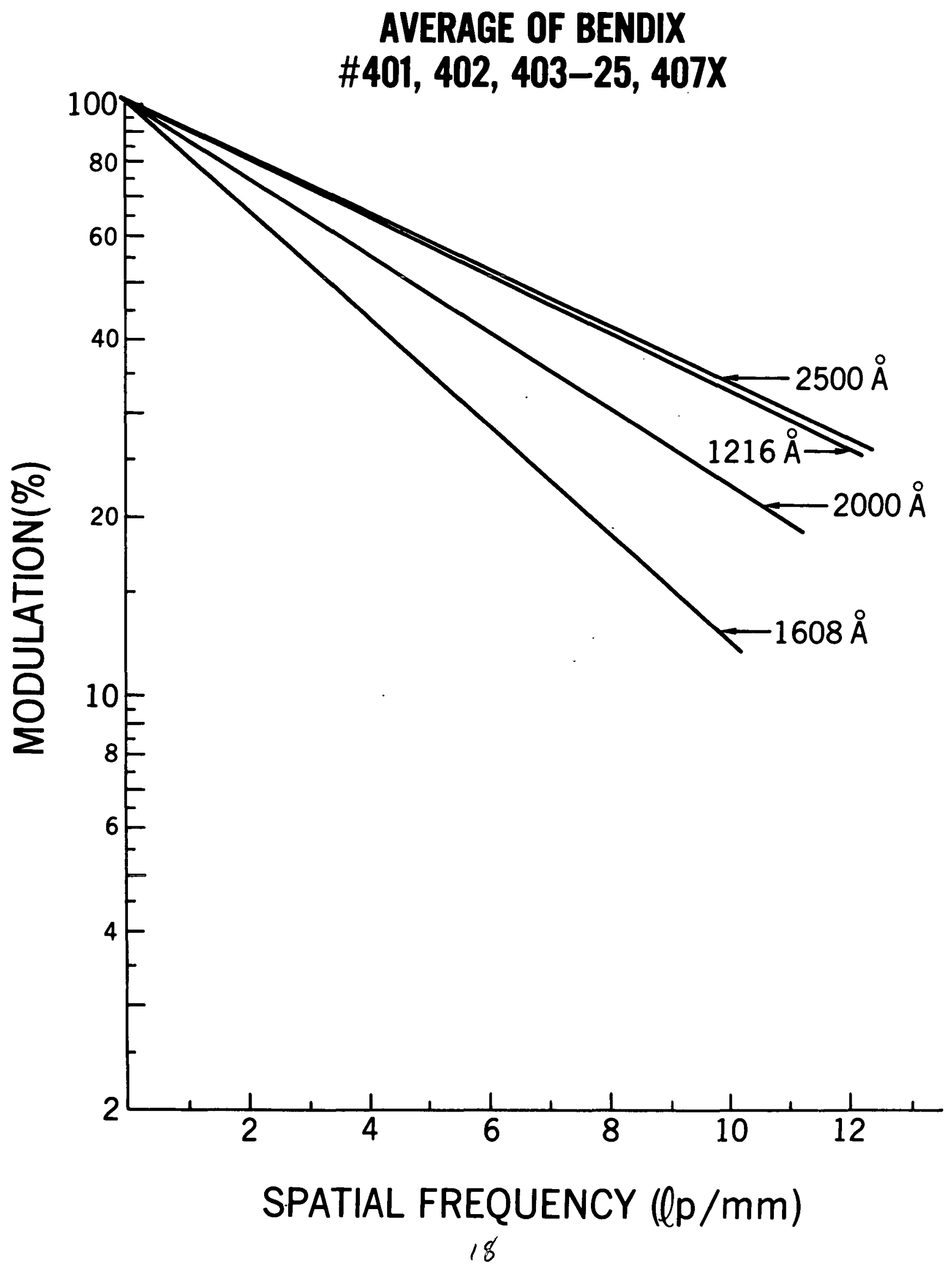




\section{AVERAGE FOR ALL CONVERTERS}

AT $5 \mathrm{lp} / \mathrm{mm}$

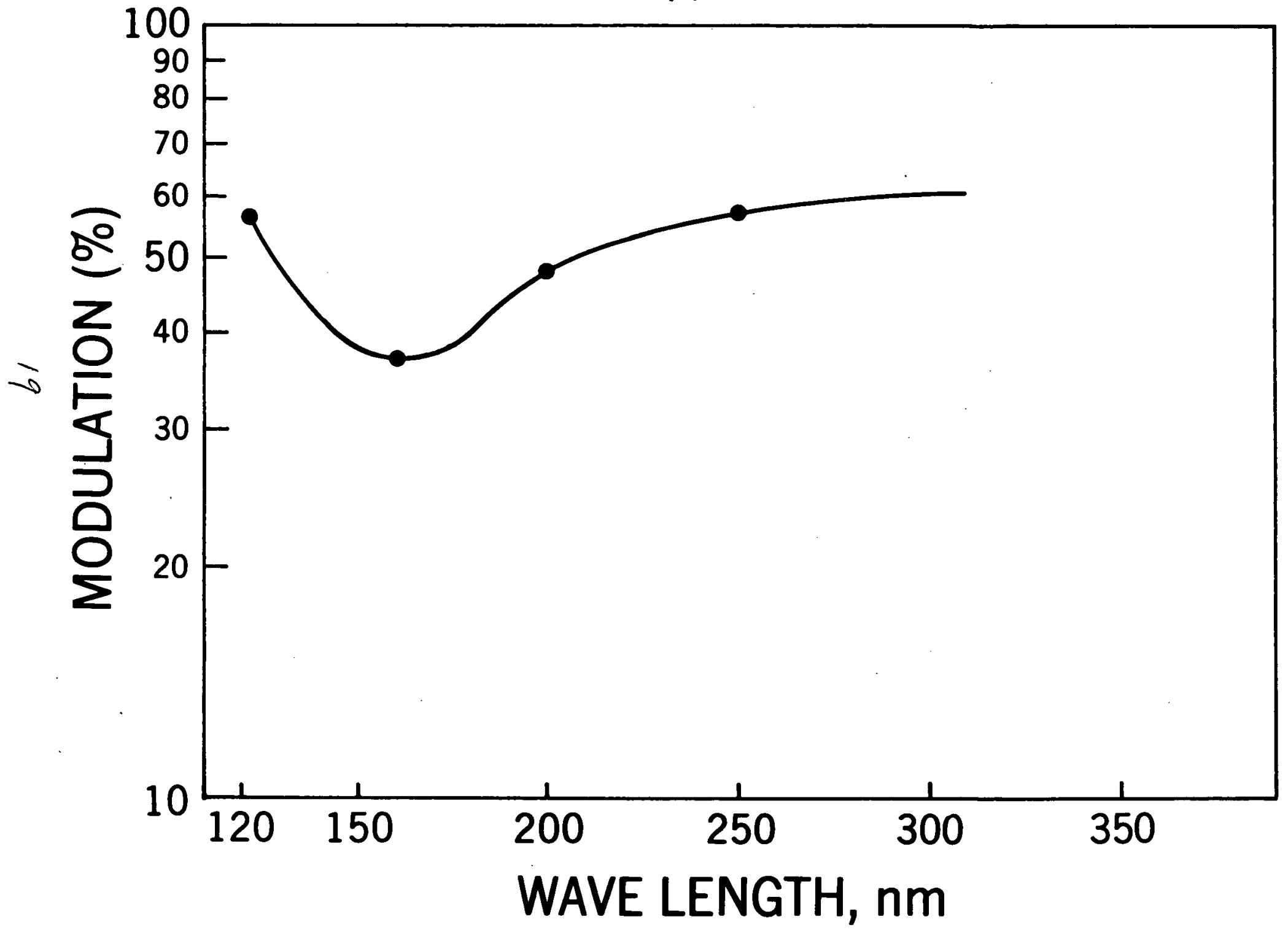




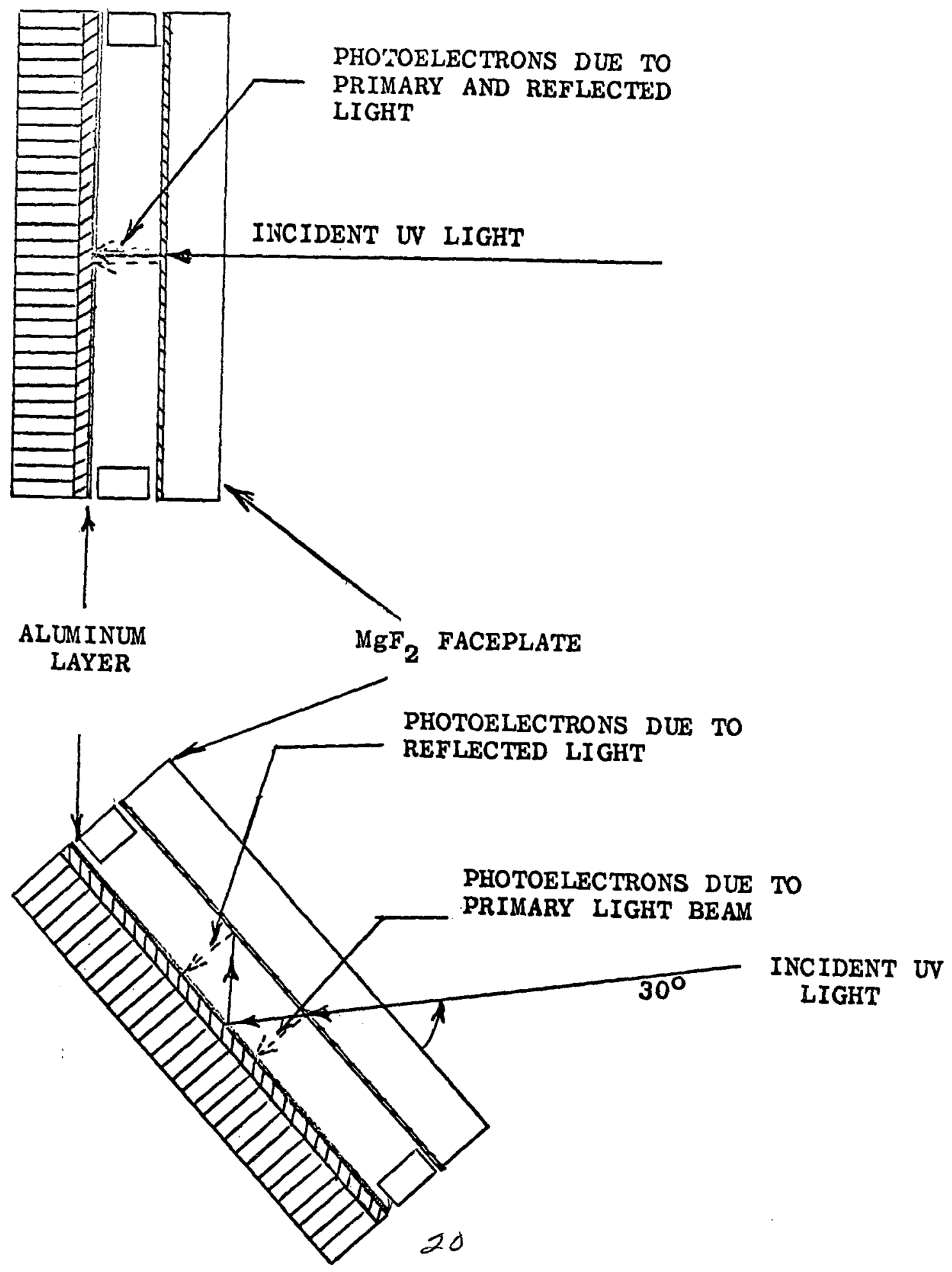




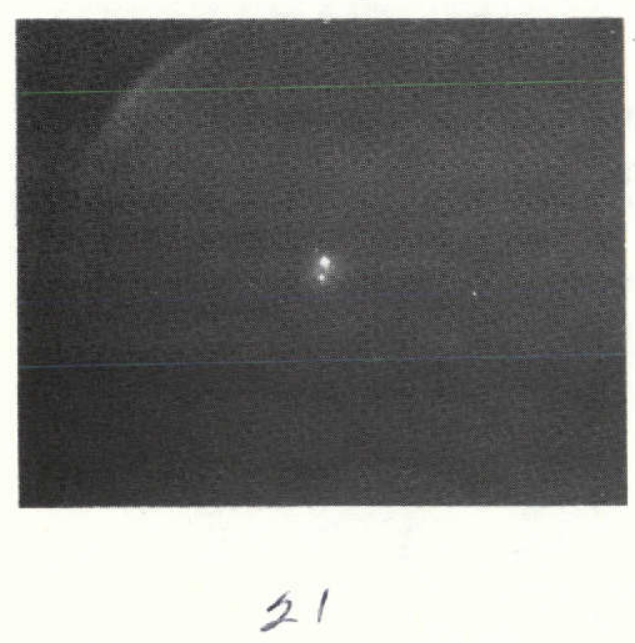




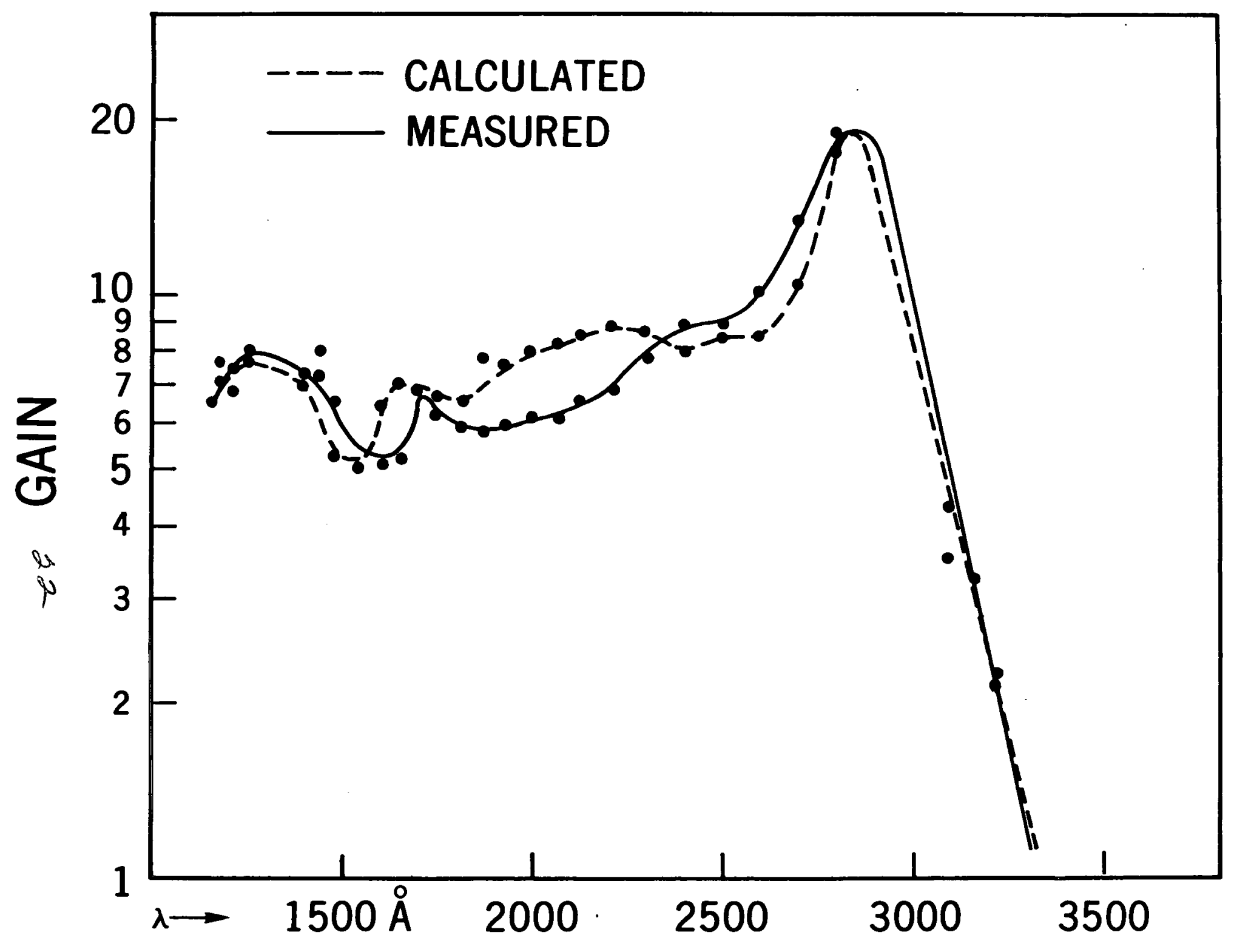






24 


\section{RANDOM VIBRATION}

FREQUENCY $(\mathrm{Hz})$ PSD ACCELERATION DURATION
$20-2000$
$.05 \mathrm{~g}^{2} / \mathrm{Hz}$
10 grms
$1 \mathrm{~min} / \mathrm{axis}$

\section{SINE VIBRATION}

ONE SWEEP (LOW TO HIGH) IN EACH PRINCIPAL AXIS \& TO THE FOLLOWING LEVELS:

FREQUENCY $(\mathrm{Hz})$ AMPLITUDE SWEEP RATE

\begin{tabular}{|c|c|c|}
\hline $5-7.5$ & 0.8 IN DA & \\
$7.5-10$ & $2.3 \mathrm{~g}$ & \\
$10-13$ & $3.0 \mathrm{~g}$ & 6 OCT /MIN \\
$13-23$ & $8.0 \mathrm{~g}$ & \\
$23-100$ & $12.0 \mathrm{~g}$ & \\
$100-200$ & $15.0 \mathrm{~g}$ & \\
\hline
\end{tabular}




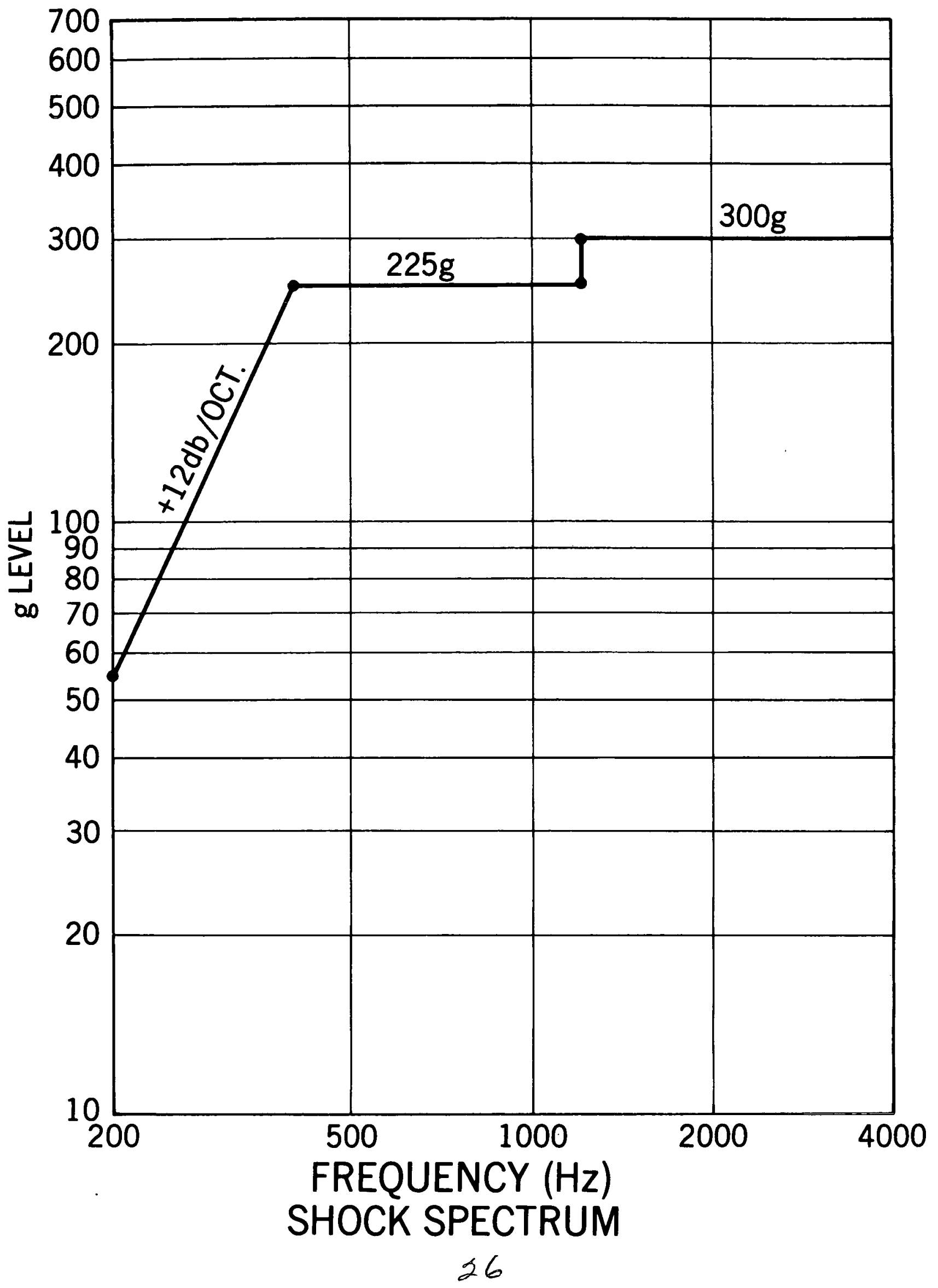

